\title{
Rugged HBT Class-C Power Amplifiers with Base-Emitter Clamping
}

\author{
Xi Luo, Subrata Halder, and James C.M. Hwang \\ Lehigh University, Bethlehem, PA 18015, USA.
}

\begin{abstract}
The ruggedness of HBT Class-C power amplifiers was improved by adding an anti-parallel diode to the amplifier input to limit the negative swing of the base-emitter voltage. The improved amplifier could withstand 3:1 instead of 2:1 mismatch in CW operation, and 2.5:1 instead of 1.5:1 mismatch in pulse operation. In contrast to other approaches with emitter ballast, active feedback, or electrostatic discharge protection circuits, the present approach is simple to implement and has negligible impact on overall amplifier output power, gain or efficiency.

Index Terms - Avalanche breakdown, heterojunction bipolar transistors, impedance matching, power bipolar transistor amplifiers, standing wave measurements, robustness
\end{abstract}

\section{INTRODUCTION}

Most power amplifiers must be sufficiently rugged to withstand output mismatch when antenna impedance changes with the environment, for example [1]. Ruggedness is especially critical to Class-C power amplifiers, for which electric breakdown is always a concern since Class-C power amplifiers were first made of vacuum tubes. To improve the ruggedness of HBT power amplifiers, several approaches have been proposed including adding an emitter ballast resistor [2], or electrostatic discharge protection circuit to the amplifier input [3], as well as using a collector voltage sensing circuit to adjust the input signal [4], the collector bias voltage [5], [6] or current [7]. However, these approaches tend to degrade the amplifier gain and output power, add circuit complexity and power consumption, and are slow to respond in the case of digital feedback. This paper proposes a different approach by adding an anti-parallel diode to the HBT base-emitter junction that is simple to implement and does not sacrifice the overall amplifier performance.

\section{EXPERIMENTAL}

The proposed approach was demonstrated monolithically on-chip in $n-p-n$ InGaP/GaAs HBT technology through a commercial foundry [8]. The HBTs typically exhibit a forward current cut-off frequency $f_{T}$ of $40 \mathrm{GHz}$, a maximum frequency of oscillation $f_{M A X}$ of $60 \mathrm{GHz}$, an open-emitter collector-base breakdown voltage $B V_{C B O}$ of $30 \mathrm{~V}$, an open-base collectoremitter breakdown voltage $B V_{C E O}$ of $15 \mathrm{~V}$, and an opencollector base-emitter breakdown voltage $B V_{B E O}$ of $7 \mathrm{~V}$.

In HBT Class-C amplifiers, the base-emitter voltage $V_{B E}$ can be the most negative when the collector-emitter voltage $V_{C E}$ is the most positive to cause collector-base avalanche breakdown [9]. By limiting the negative swing of $V_{B E}$, breakdown can be prevented and the amplifier can be more rugged. Fig. 1 shows three types of Class-C amplifier demonstrated with an HBT of

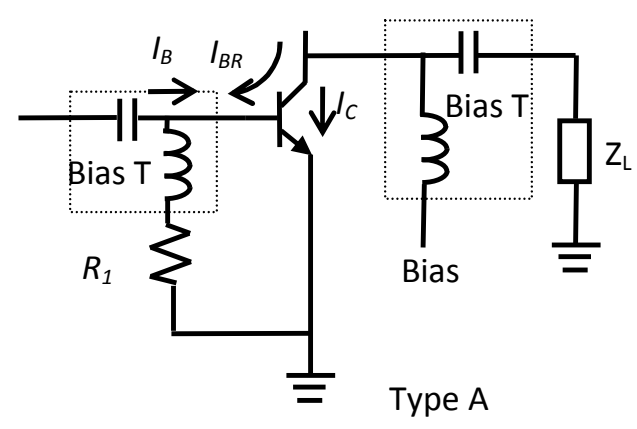

(a)

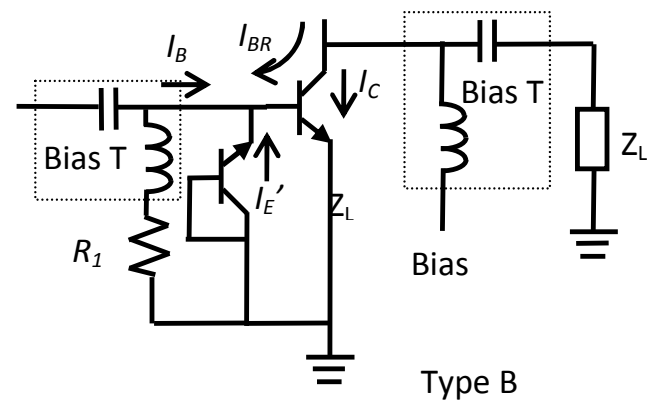

(b)

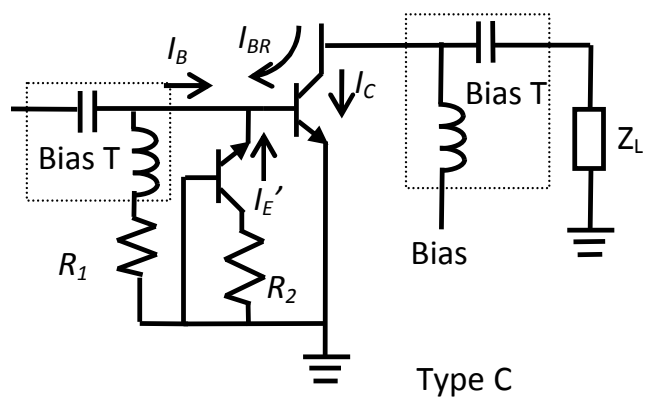

(c)

Fig. 1. Three types of HBT Class-C power amplifiers (a) without base-emitter clamping, (b) with base-emitter clamping, and (c) with base-emitter clamping enhanced by a series resistor $R_{2}$. The main HBT is self biased with resistor $R_{2}$ between the base and the emitter.

eight emitter fingers, each finger $2-\mu \mathrm{m}$ wide and $20-\mu \mathrm{m}$ long.

The HBT is self biased with a $50-\Omega$ resistor $R_{1}$ between the base and emitter. In addition, in the Type-B and Type-C amplifiers, the based-emitter junction is shunted by a singlefinger HBT of $2 \mu \mathrm{m} \times 20 \mu \mathrm{m}$. The single-finger HBT, with its base shorted to the collector, serves as an anti-parallel diode to limit the negative swing of $V_{B E}$ of the main HBT. Finally, in the Type-C amplifier, a series resistance $R_{2}$ of $100 \Omega$ is added 


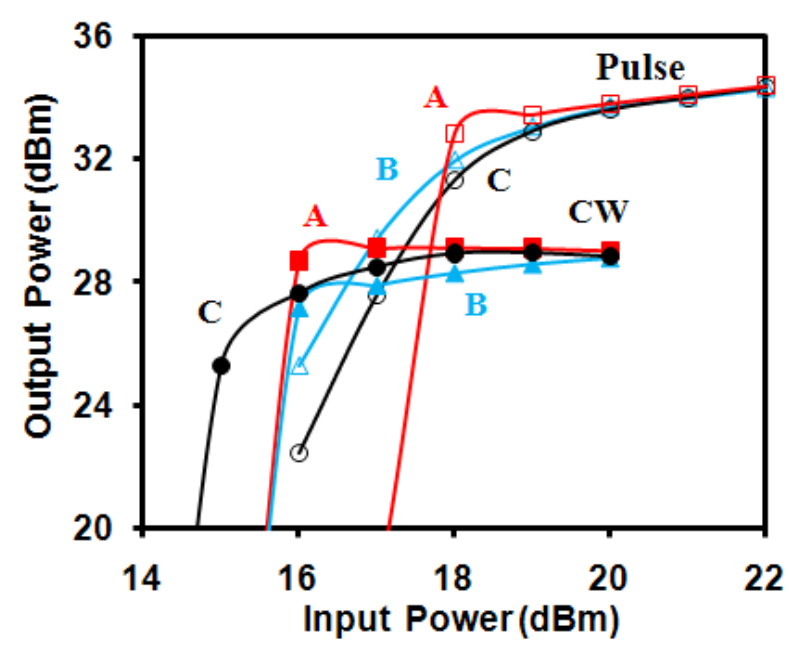

(a)

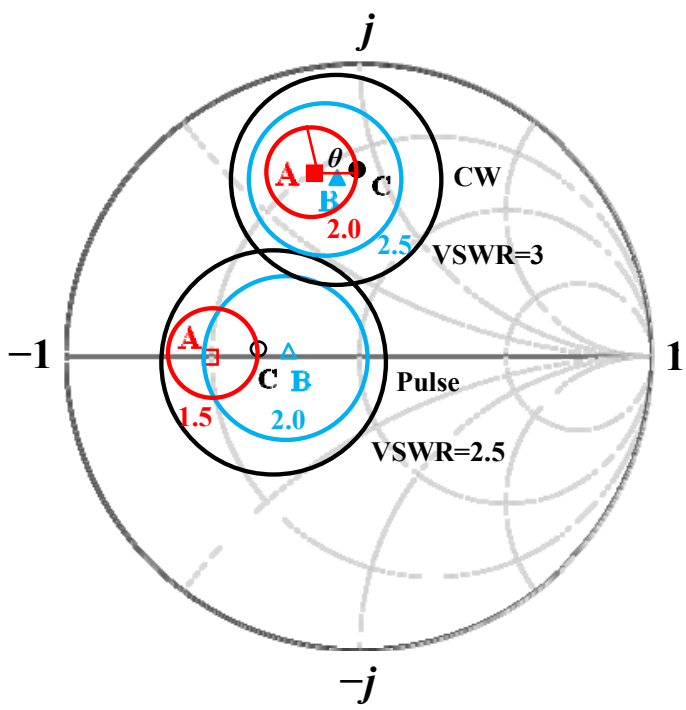

(b)

Fig. 2. (a) Power-sweep characteristics and (b) optimum load impedances of $(\square, \square)$ Type-A, $(\Delta, \Delta)$ Type-B, and $(\bullet, \circ)$ Type-C amplifiers in (filled symbols) $\mathrm{CW}$ and (empty symbols) pulse operations. $V_{C E}=12 \mathrm{~V}$. $\theta$ represents mismatch angle. Maximum VSWR circles the amplifiers can withstand without burn out are plotted around the optimum loads.

to the collector of the single-finger HBT to help drive it further into saturation under negative $V_{B E}$.

In avalanche breakdown, holes generated in the collector can flow to the base $\left(I_{B R}\right)$ and cause positive feedback and burn out. The base-emitter clamping diode in the Type-B or Type-C amplifiers can not only reduce the maximum collector-base voltage $V_{C B}$, but also draws current $I_{E}$ ' into the base to compensate $I_{B R}$, thereby reducing the impact of avalanche breakdown.

The amplifiers were characterized at $6 \mathrm{GHz}$ in both $\mathrm{CW}$ and pulse operations by using a Maury automated load-pull system. In pulse operation, both $V_{C E}$ and RF input power $P_{I N}$ were turned on for only $1 \mu$ s with a pulse repetition frequency

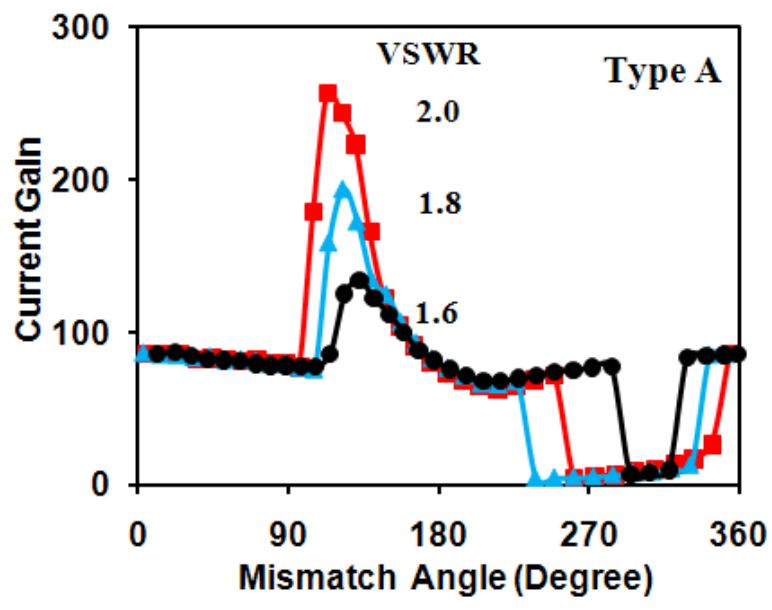

(a)

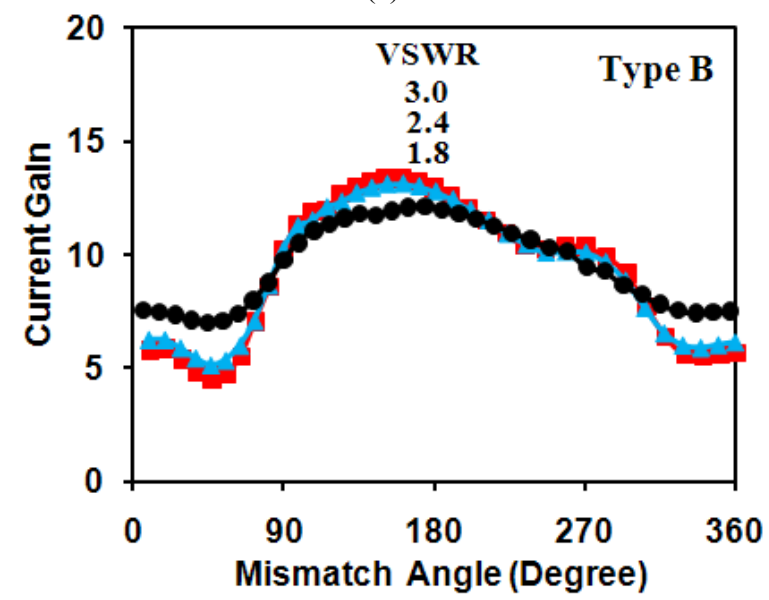

(b)

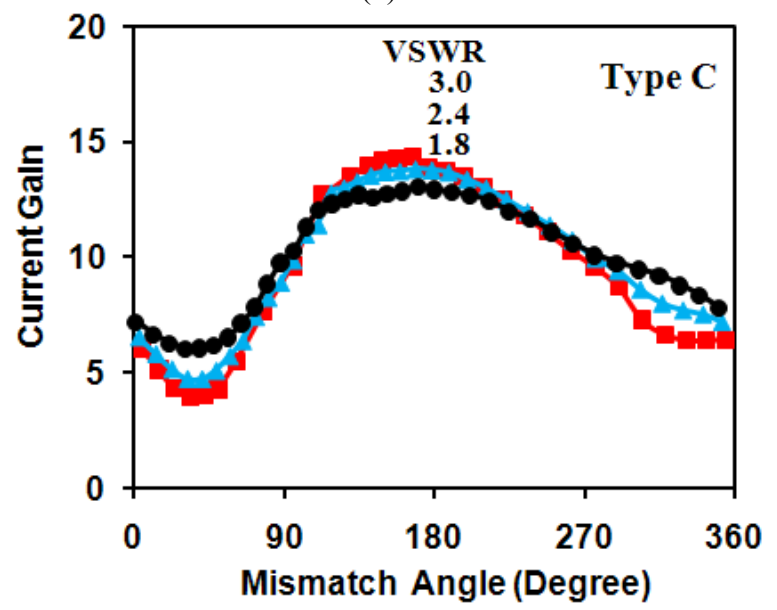

(c)

Fig. 3. DC forward current gain for (a) Type-A, (b) Type-B, and (c) Type-C amplifiers in $\mathrm{CW}$ operation under different mismatch magnitude and angles. $V_{C E}=12 \mathrm{~V}$.

of $2.78 \mathrm{kHz}$ to minimize self heating. The RF output power was sampled in the middle of the pulse. The average collector current was sensed by an oscilloscope via a $5-\Omega$ series resistor. In addition to power-sweep measurement in the frequency domain, time-domain current and voltage waveforms with 


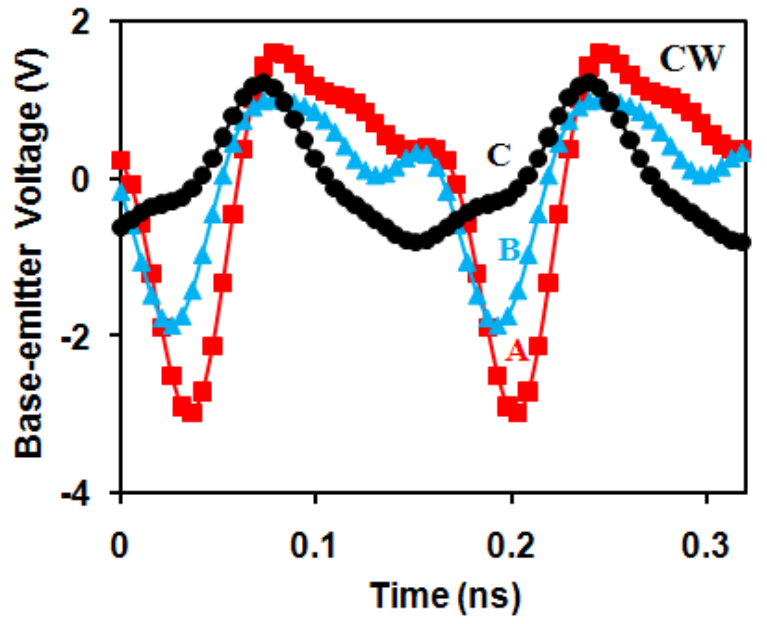

(a)

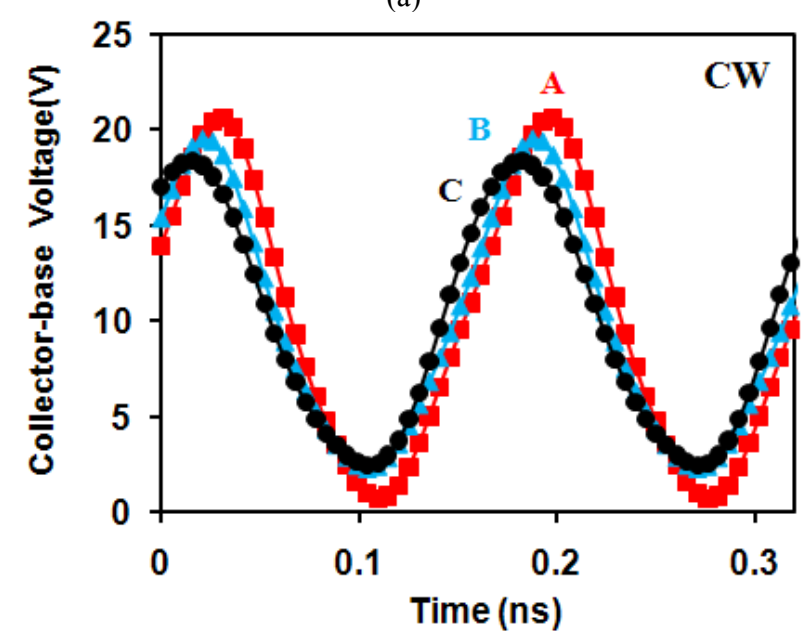

(b)

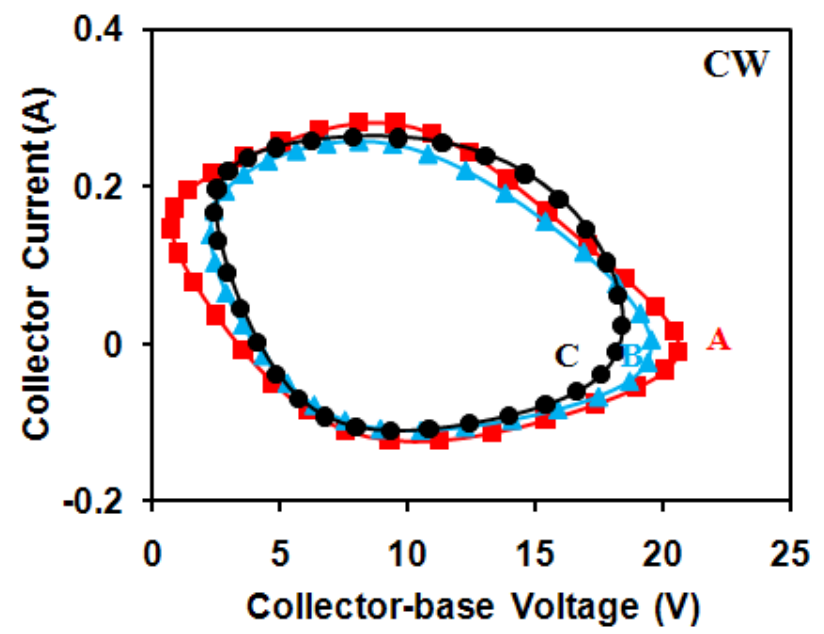

(c)

Fig. 4. (a) Base-emitter voltage waveform, (b) collector-base voltage waveform, and (c) collector dynamic load lines of ( $\square$ ) Type$\mathrm{A},(\Delta)$ Type-B, and $(\bullet)$ Type- $\mathrm{C}$ amplifiers in $\mathrm{CW}$ operation. $V_{C E}=$ 12 V. $P_{I N}=14 \mathrm{dBm} . Z_{L}=14+j 40 \Omega$.

three harmonics were measured at the base and collector by using an HP 71500A microwave transition analyzer [10].

\section{POWER-SWEEP CHARACTERISTICS}

With $V_{C E}=12 \mathrm{~V}$ and optimum source and load matches, the amplifiers delivered twice the output power in pulse operation than in $\mathrm{CW}$ operation. Fig. 2 shows that in CW operation, all three types of amplifier delivered $29-\mathrm{dBm}$ output under optimum loads $Z_{L}$ of $22+j 44 \Omega, 24+j 44 \Omega$, and $25+j 50 \Omega$ for the Type-A, Type-B, and Type-C amplifiers, respectively. This shows that the modification for base-emitter clamping does not affect the amplifier output significantly. Similarly, in pulse operation all three types of amplifier delivered 34-dBm output, but the optimum loads became essentially real at $24 \Omega$, $35 \Omega$, and $37 \Omega$ for the Type-A, Type-B, and Type-C amplifiers, respectively. The improvement in output power could be attributed to reduced Kirk effect and avalanche breakdown in pulse operation.

The amplifiers with base-emitter clamping were more rugged against load mismatch as determined by keeping the source impedance optimum while varying the load impedance for different voltage standing-wave ratios (VSWRs). It was found that in $\mathrm{CW}$ operation and under $V_{C E}=13 \mathrm{~V}$ and $P_{I N}=$ $15 \mathrm{dBm}$, the maximum VSWRs the Type-A, Type-B, and Type-C amplifiers could withstand were 2.0, 2.5, and 3.0, respectively. Similarly, in pulse operation and under $V_{C E}=10$ $\mathrm{V}$ and $P_{I N}=19 \mathrm{dBm}$, the maximum VSWRs were 1.5, 2.0 and 2.5 for the Type-A, Type-B, and Type-C amplifiers, respectively. The maximum VSWR circles were plotted around the optimum loads in Fig. 2(b).

Avalanche breakdown depends on not only the magnitude but also the angle $\theta$ of load mismatch. Fig. 3 shows the DC forward current gain in $\mathrm{CW}$ operation under different mismatch magnitudes and angles with $V_{C E}=12 \mathrm{~V}$ and $P_{I N}=$ $14 \mathrm{dBm}$. It can be seen that for the Type-A amplifier, the current gain peaks between $110^{\circ}$ and $160^{\circ}$ of mismatch when the breakdown-generated electron current adds to the collector current while the breakdown-generated hole current subtracts from the base current. By contrast, the current gain of the Type-B or Type-C amplifier does not increase significantly with either the magnitude or angle of mismatch, indicating that avalanche breakdown is successfully suppressed.

\section{CURRENT AND VOLTAGE WAVEFORMS}

Because the current gain was difficult to measure directly in pulse operation, base and collector waveforms were measured to confirm the suppression of avalanche breakdown. Fig. 4 shows the base-emitter voltage waveform, collector-base voltage waveform, and dynamic load lines in $\mathrm{CW}$ operation with $V_{C E}=12 \mathrm{~V}, P_{I N}=14 \mathrm{dBm}$ and $Z_{L}=14+j 40 \Omega$ near where the current gain peaks. It can be seen that the most negative $V_{B E}$ 's were approximately $-3 \mathrm{~V},-2 \mathrm{~V}$ and $-1 \mathrm{~V}$ for the Type-A, Type-B and Type-C amplifiers, respectively. Meanwhile, the most positive $V_{C E}$ 's were $21 \mathrm{~V}, 19 \mathrm{~V}$ and 18 $\mathrm{V}$, for the Type-A, Type-B and Type-C amplifiers, respectively. The dynamic load lines confirm that the Type-A amplifier had the highest peak collector current and voltage. 


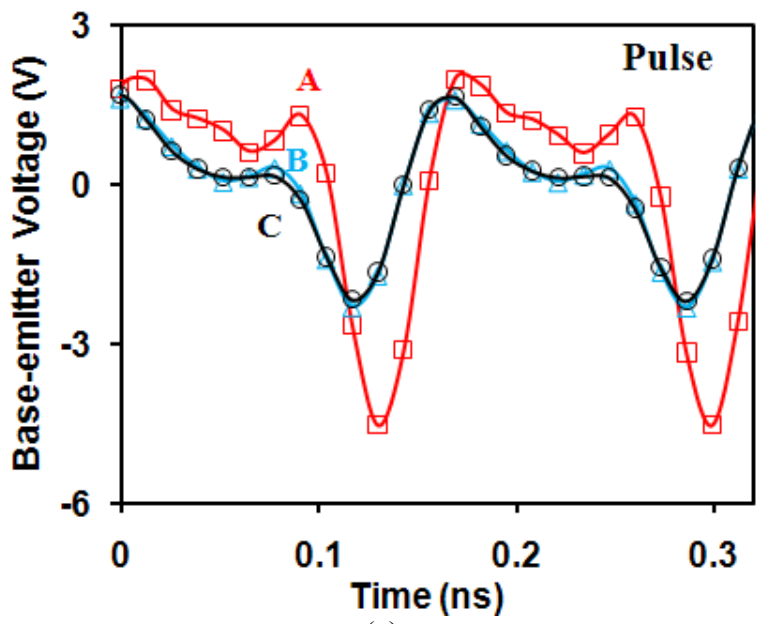

(a)

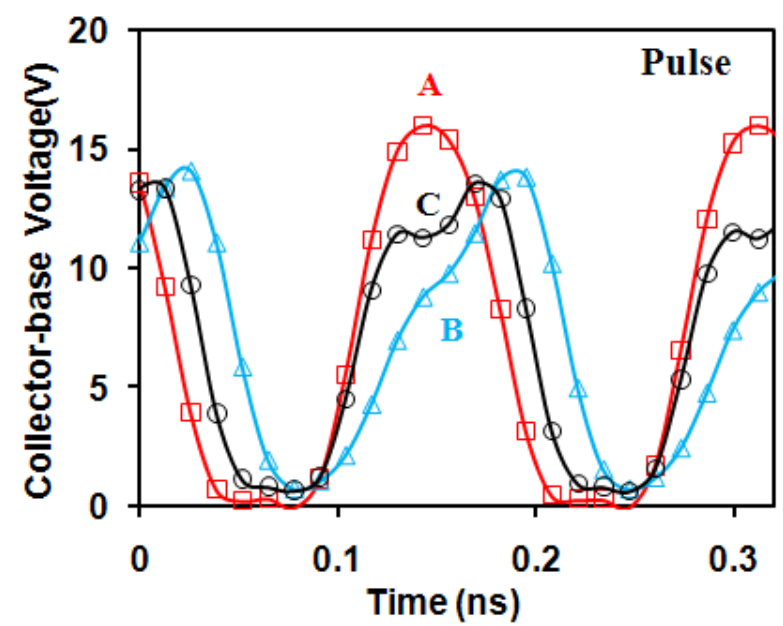

(b)

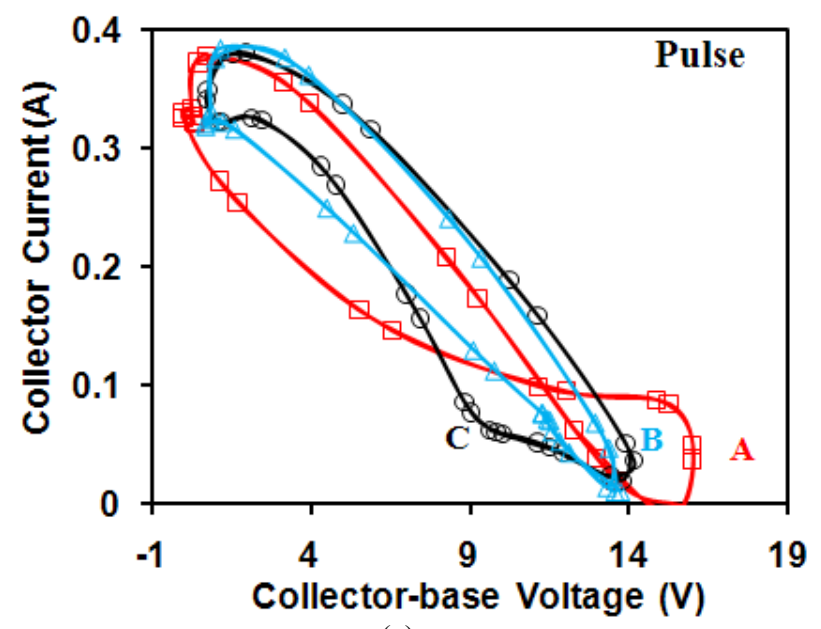

(c)

Fig. 5. (a) Base-emitter voltage waveform, (b) collector-base voltage waveform, and (c) collector dynamic load lines of ( $\square$ ) TypeA, $(\triangle)$ Type-B, and (०) Type-C amplifiers in pulse operation. $V_{C E}=$ $10 \mathrm{~V} . P_{I N}=19 \mathrm{dBm} . Z_{L}=42+j 10 \Omega$.

The waveforms measured in pulse operation with $V_{C E}=10 \mathrm{~V}$, $P_{I N}=19 \mathrm{dBm}$ and $Z_{L}=42+j 10 \Omega$ exhibited the same trend as seen in Fig. 5.

\section{CONCLUSION}

With base-emitter clamping on the input of HBT Class-C power amplifiers, avalanche breakdown as well as the impact of breakdown-induced hole current was suppressed. The improved amplifier could withstand higher load mismatch without sacrificing the overall amplifier output power, gain and efficiency.

\section{REFERENCES}

[1] A. Keerti, and A. V. H. Pham, "RF characterization of SiGe HBT power amplifiers under load mismatch," IEEE Trans. Microwave Theory Techniques, vol. 55, pp. 207-214, Feb. 2007.

[2] A. Scuderi, A. Scuderi, F. Carrara, and G. Palmisano, "A VSWR-rugged silicon bipolar RF power amplifier," in Proc. IEEE Bipolar/BiCMOS Circuits Technology Meet., Oct. 2005, pp. 116-119.

[3] D. A. Teeter, K. Muhonen, D. Widay, and M. Fresina, "GaAs HBT ESD diode layout and its relationship to human body model rating," in Proc. IEEE Bipolar/BiCMOS Circuits Technology Meet., Sep. 2006, pp. 1-4.

[4] K. Yamamoto, S. Suzuki, K. Mori, and et al., "A 3.2-V operation single-chip dual-band $\mathrm{AlGaAs} / \mathrm{GaAs}$ HBT MMIC power amplifier with active feedback circuit technique," IEEE $J$. Solid-State Circuits, vol. 35 , pp. 1109-1120, Aug. 2000.

[5] A. Bezooijen, F. V. Straten, R. Mahmoudi, and A. H. M. V. Roermund, "Power amplifier protection by adaptive output power control," IEEE J. Solid-State Circuits, vol. 42, pp. 18341841, Sep. 2007.

[6] H. Shin, H. Ju, M. F. Chang, K. Nellis, and P. Zampardi, "An output VSWR protection Circuit using collector/emitter avalanche breakdown for SiGe HBT power amplifiers," IEICE Trans. Electronics, vol. E87-C, pp. 1643-1645, Sep. 2004.

[7] W. Karoui, and T. Parra, "A protection circuit for HBT RF power amplifier under load mismatch conditions," in Dig. Workshop Circuits Systems TAISA Conf., Jun. 2008, pp. 241-244.

[8] HBT H02U-10 technology, WIN Semiconductors, Taoyuan, Taiwan.

[9] M. Rudolph, F. Schnieder, and W. Heinrich, "Modeling emitter breakdown in GaAs-based HBTs," IEEE MTT-S Int. Microwave Symp. Dig., Jun. 2003, pp. 651-654.

[10] C. J. Wei, Y. E. Lan, and J. C. M. Hwang, "Waveform-based modeling and characterization of microwave power heterojunction bipolar transistors," IEEE Trans. Microwave Theory Techniques, vol. 43, pp. 2899-2903, Dec. 1995. 